\title{
Acoustically enhanced microfluidic mixer to synthesize highly uniform nanodrugs without the addition of stabilizers
}

This article was published in the following Dove Press journal: International Journal of Nanomedicine

\author{
Nguyen Hoai An Le' \\ Hoang Van Phan' \\ Jiaqi $Y^{2}$ \\ Hak-Kim Chan ${ }^{2}$ \\ Adrian Neild' \\ Tuncay Alan' \\ 'Department of Mechanical and \\ Aerospace Engineering, Monash \\ University, Melbourne, VIC, ${ }^{2}$ The \\ Advanced Drug Delivery Group, \\ Faculty of Pharmacy, University of \\ Sydney, Sydney, NSW, Australia
}

Background: This article presents an acoustically enhanced microfluidic mixer to generate highly uniform and ultra-fine nanoparticles, offering significant advantages over conventional liquid antisolvent techniques.

Methods: The method employed a 3D microfluidic geometry whereby two different phases solvent and antisolvent - were introduced at either side of a $1 \mu \mathrm{m}$ thick resonating membrane, which contained a through-hole. The vibration of the membrane rapidly and efficiently mixed the two phases, at the location of the hole, leading to the formation of nanoparticles.

Results: The versatility of the device was demonstrated by synthesizing budesonide (a common asthma drug) with a mean diameter of $135.7 \mathrm{~nm}$ and a polydispersity index of 0.044 .

Conclusion: The method offers a 40-fold reduction in the size of synthesized particles combined with a substantial improvement in uniformity, achieved without the need of stabilizers.

Keywords: microfluidics, nanodrugs, budesonide, liquid antisolvent method

\section{Introduction}

Approximately $40 \%$ of drugs used in the pharmaceutical industry are poorly soluble, which results in poor bioavailability and uncontrollable precipitation after dosing and requires employment of extreme basic/acidic conditions to enhance solubility. ${ }^{1}$ The drug's dissolution rate can be increased proportionally by reducing its size to nanometer scales, hence increasing its surface area-to-volume ratio. ${ }^{2-4}$ Using nanoscale drugs also provides a wide range of therapeutic advantages, ${ }^{5-11}$ enhances targeted delivery to specific tissues, and improves the effectiveness of the drug by lowering its resistance and dose requirement. ${ }^{12,13}$ In aerosol drug delivery, particle size plays a critical role in determining how deep the drugs will be deposited, ${ }^{14,15}$ hence providing a crucial advantage to treat lung diseases. ${ }^{16-21}$

There are two common approaches to synthesize nanodrugs. The first of these, the attrition method, is a "top-down" approach, which relies on breaking down the bulk structure of the drug into finer sizes. ${ }^{22}$ The method is capable of generating nanodrugs without employing harsh solvents; ${ }^{23}$ however, it requires a significant amount of energy to reduce the sizes to submicron levels and hence generates a high level of heating during operation, which may alter the physical state of drugs and surfactants. ${ }^{24}$ The alternative "bottom-up" techniques aim to synthesize the drugs from smaller elements. ${ }^{25-27}$ The widely used liquid antisolvent (LAS) method offers relative simplicity by combining room temperature operation with low pressure requirements (in contrast to other methods such as supercritical antisolvent method, ${ }^{28}$ where high
Correspondence: Tuncay Alan Department of Mechanical and Aerospace Engineering, Monash University, 19 College Walk, Clayton Campus, Wellington Road Clayton, VIC 3800, Australia Email tuncay.alan@monash.edu 
pressures are required). ${ }^{23}$ The LAS procedure is solely based on the nucleation of the drug when two different liquid phases are mixed. One phase is the solvent phase, where the drug of interest is dissolved. The addition of antisolvent phase into the solvent creates a supersaturated concentration environment, which affects the nucleation rate of the particles.

Typically, higher nucleation rates result in a larger proportion of submicrometer sized particles. However, during the mixing, the nucleation rate competes with the condensation and coagulation of the particles, which in turn work to reduce the supersaturation condition, hence increasing the particle size. Damkoehler number, $\mathrm{Da}=t_{\text {mix }} / t_{\text {precipitation }}$ (where $t_{\text {mix }}$ is the mixing time and $t_{\text {precipitation }}$ is the precipitation time), affects the nucleation rate, and the larger Da values result in larger particle sizes. ${ }^{29}$ Particle size can be reduced by increasing $t_{\text {precipitation }}$ often by means of additives and stabilizers, ${ }^{30}$ which coat the nanoparticle surface to prevent unwanted growth of the drug in suspension. ${ }^{31}$ Size reduction can also be achieved by reducing the mixing time $t_{\text {mix }}$ and delaying precipitation through mechanical means. Here, we demonstrate an acoustically enhanced microfluidic method to significantly reduce the mixing time (down to $3 \mathrm{~ms}$ ), and synthesize highly uniform, ultrafine particles without the use of stabilizers. Avoiding stabilizers is particularly desirable as their addition during production can cause toxicity issues and undermine the therapeutic efficiency of the drug. ${ }^{24}$ Moreover, selecting the optimal surfactants is often burdensome, as the interaction between drugs and added emulsions is poorly understood at nanoscales.

Central to the approach is a vibrating membrane, which is embedded in a microfluidic channel. Under acoustic excitation, the interaction of the resonating membrane with the surrounding fluid media generates an acoustic streaming field and corresponding very strong vortices. ${ }^{32}$ As a result, the antisolvent and solvent phases are rapidly and efficiently mixed creating the necessary supersaturation condition for the drugs with ultrafine particle sizes to nucleate while preventing accumulation of the formed particles. The commonly used asthma medication budesonide was chosen as a suitably well-characterized test case for the experiments aimed at demonstrating performance of the method. ${ }^{33-36}$ Budesonide is one of the most valuable therapeutic agents to treat pulmonary diseases yet its low aqueous solubility limits its absorption after oral delivery and poses a challenge for its efficacy. Generating a monodisperse suspension of submicrometer-diameter budesonide particles can address this issue..$^{37,38}$ Numerous other methods were previously employed to synthesize budesonide nanodrugs. For instance, oil-in-water $(\mathrm{O} / \mathrm{W})$ emulsion evaporation techniques resulted in $200 \mathrm{~nm}$ diameter particles in the presence of surfactants. ${ }^{39}$ $640 \mathrm{~nm}$ diameter particles were possible without surfactants by using a high-pressure homogenizing method, at 60 cycles and $<1,000$ bars of pressure. ${ }^{40}$ The technique presented here combines operational simplicity with small diameter nanoparticles while avoiding the use of stabilizers, hence it offers a significant advantage over the earlier approaches.

\section{Methods}

\section{Microfluidic device preparation}

The device consists of a $500 \mu \mathrm{m}$ wide, $50 \mu \mathrm{m}$ deep polydimethylsiloxane (PDMS) microfluidic channel assembled with a free standing, 1,000 nm thick silicon nitride membrane (Figure 1A), through which a hole is etched. For the membranes, the front-side geometric features and back-side openings are patterned on $1 \mu \mathrm{m}$ thick silicon nitride (SiN)-coated (100)-oriented Si wafers (4D LABS, Burnaby, BC, Canada) using a combination of photolithography and reactive ion etching (RIE). The wafers are then immersed in a $5 \mathrm{M}$ potassium hydroxide $(\mathrm{KOH})$ solution at $65^{\circ} \mathrm{C}$ for $\sim 15$ hours to selectively etch the $\mathrm{Si}$ and release the free-standing $\mathrm{SiN}$ membranes.

The PDMS channels are fabricated using standard procedures. After a mold of the channel geometry is etched by deep RIE (DRIE) into a Si wafer, its surface is rendered hydrophobic by a layer of Teflon coating. A PDMS solution mixed with curing agent, SYLGARD ${ }^{\circledR} 184$ (Dow Corning, MI, USA) at ratio 10:1 (w/w), is cast on the mold. The mixture is left in a vacuum pump for 2 hours and then on a hot plate at $65^{\circ} \mathrm{C}$ for complete curing.

To assemble the devices, the PDMS channels are cut and bonded onto the chips containing individual membranes. The entire chip is then adhered onto a glass microscope slide, as shown in Figure 1B. A tube connector attached to the underside of the membrane (Figure 1C) allows a solution of diluted drugs to access the microfluidic channel.

The operation of the micromixer is vastly different when the piezoelectric transducer (PZT) is turned on and off, as shown in Figure 1D. In the absence of acoustic excitation, the fluids are separated within the channel with a clearly defined boundary. Once the device is activated, this boundary is replaced with the mixed solution, which covers the width of the channel; the device generates vortices around the membrane to rapidly mix the solution.

\section{Materials}

Budesonide was supplied by Yicheng Chemical Corporation (Jiangsu, China). Ethanol (99.7\%, v/v; Merck Millipore, 
A

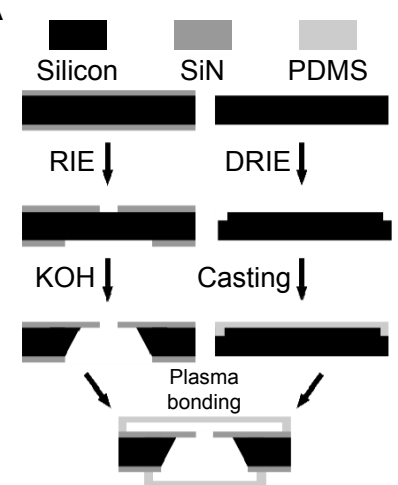

B
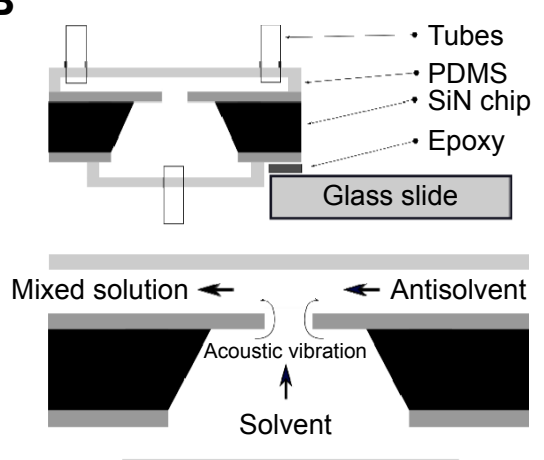

C

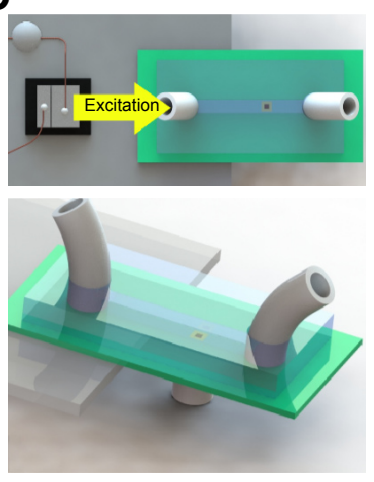

D
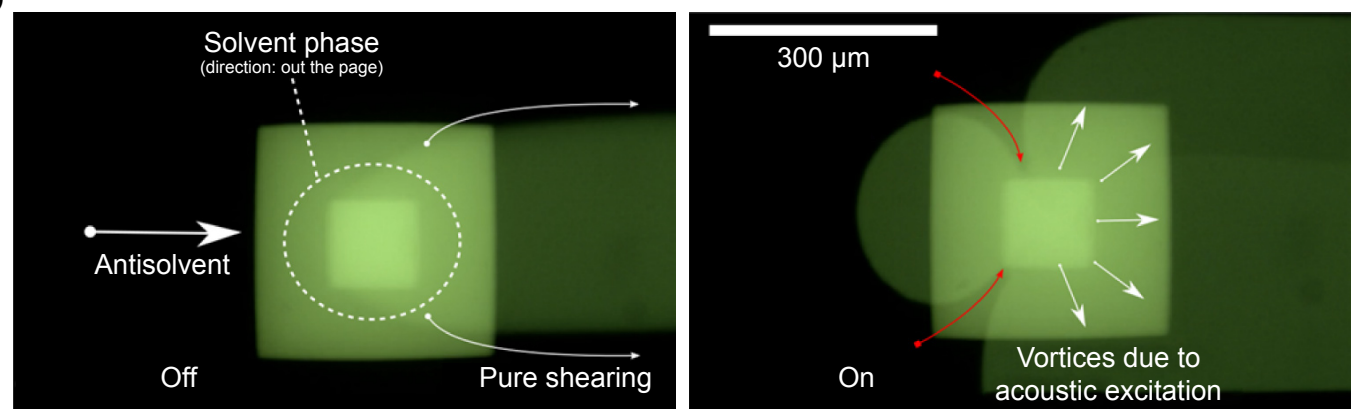

Figure I Device schematic and operation.

Notes: (A) Schematic representation of the micromixer device; the solvent is fed from the bottom side of the silicon nitride while antisolvent phase is introduced in the top layer. (B) A simplified summary of the fabrication steps, showing how the channel and the membranes are fabricated with RIE, KOH etching, and DRIE. (C) A Solidworks 3D model, showing how the piezoelectric disk is assembled into the device, and (D) experimental snapshots demonstrating the mixing of two liquids before and after acoustic actuations (fluorescent dye was used to enhance visualization).

Abbreviations: DRIE, deep reactive ion etching; $\mathrm{KOH}$, potassium hydroxide; RIE, reactive ion etching; PDMS, polydimethylsiloxane.

Billerica, MA, USA) was employed as the principal solvent for the poorly dissolved drug. Purified water (Milli-Q ${ }^{\circledR}$; Millipore Merck, Darmstadt, Germany) was used as the antisolvent for the system. Throughout the experiments, the ratio between solvent and antisolvent was kept constant at $1: 4$.

\section{Experimental procedures - particle preparation and characterization}

During the experiments, the membrane was excited at its resonant frequency (ranging from 100 to $300 \mathrm{kHz}$ depending on the geometries used) by a PZT glued onto the glass slide. The PZT was driven by a signal generator (Stanford Research Systems DS345) and an amplifier (AG 1006; T\&C Power Conversion, Inc., Rochester, NY, USA) at 200 peak-to-peak voltage (Vpp). The membrane's resonant frequency is found experimentally by frequency sweeping.

After synthesis, the size and distribution of particles were characterized through a dynamic light scattering (DLS) system (Zetasizer Nano ZS; Malvern Instruments, Malvern, UK), which surveys particles in suspension. The system analyzed the velocity distribution of particles moving by Brownian motion, providing a distribution of particle sizes (with a minimum of 10 measurements for each distribution). Typically, a solution of $400 \mu \mathrm{L}$ was examined in a narrowed cuvette. DLS results provide a $Z$-average diameter (which corresponds to the mean diameter of the particles in the nanosuspension) and a polydispersity index (PDI), a dimensionless measure of the spread within the distribution. PDI values that are close to 0 indicate monodisperse particle sizes, whereas values approaching 1 indicate a wide spread in the particle size distribution. Typically, a PDI value of $<0.05$ is considered to be highly monodisperse, ${ }^{41}$ while PDI values ranging from 0.2 to 0.5 are considered to represent a relatively narrow particle size distribution. ${ }^{3,42}$

As a final characterization step, scanning electron microscopy (SEM) was performed to determine the size and shape of the produced nanoparticles. Prior to SEM, the solution was positioned on a glass slide and liquid was allowed to evaporate at room temperature. This slide was then sputter-coated with gold for 90 seconds with a current of $25 \mathrm{~mA}$. The samples were then imaged with an FEI Nova NanoSEM 430 Instrument.

During the experiments, we investigated two different configurations. First (mode 1, Figure 2A), solvent and antisolvent were fed from the top surface of the membrane; 
A

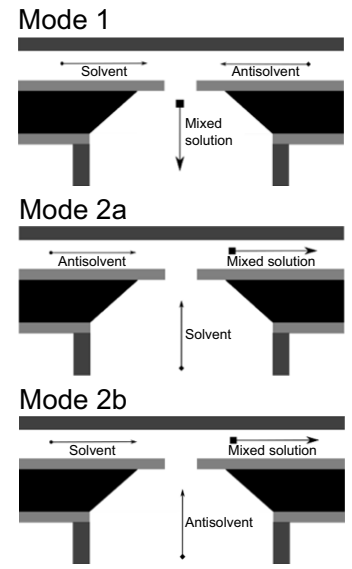

B

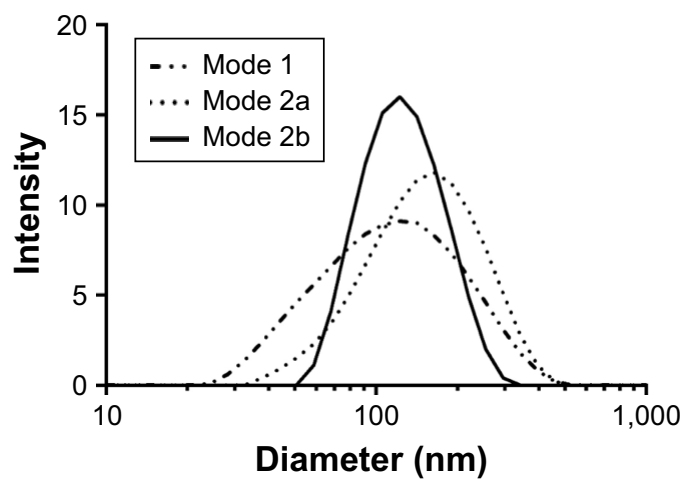

Figure 2 Comparison between different operational modes of the micromixer.

Notes: (A) Simplified schematic diagrams, and (B) DLS results, demonstrating the particle size and distribution for different modes, considering 10 measurements on $400 \mu L$ samples. The mean particle diameters and standard deviations are $99 \pm 80,136 \pm 61$, and I20 $444 \mathrm{~nm}$ for modes I, 2a, and $2 \mathrm{~b}$, respectively.

Abbreviation: DLS, dynamic light scattering.

mixing took place at the through-hole where acoustic excitation generated a strong vortex. For mode 2 operation (Figure 2A), the solution was introduced on the top channel and the antisolvent was added from the underside, via the through-hole in the membrane. In mode 2, the antisolvent was pumped from the underside in mode $2 \mathrm{a}$; in contrast, the solvent was introduced from the bottom in mode $2 b$. As shown in Figure 2B, mode $2 \mathrm{~b}$ results in the smallest mean particle diameter and the most uniform distribution of nanoparticles, hence this mode of operation was employed throughout the experiments.

\section{Results}

The comparison of the particle size distribution with and without acoustic excitation of the membrane is presented in Figure 3 , in which the flow rate was fixed at $8 \mu \mathrm{L} /$ minute, the drug concentration was $0.2 \mathrm{mg} / \mathrm{mL}$, and the membrane was actuated at a frequency of $177.6 \mathrm{kHz}$. As expected, the particle size distribution in both cases follows a log-normal distribution. ${ }^{43,44}$ Without any actuation, the passive $3 \mathrm{D}$ $\mathrm{T}$-junction proposed in this study is capable of reducing the mean size of the particles to $210.3 \pm 154.8 \mathrm{~nm}$ (based on 10 repeat measurements). This is already a 20 -fold reduction in size in comparison to other microfluidic techniques employing passive T-junction mixers, for which the average particle size was $5.85 \mu \mathrm{m}$ in the absence of stabilizing chemicals. ${ }^{45}$

After the acoustic feature of the mixer was activated, the average particle diameter was further reduced to $135.7 \mathrm{~nm}$. Moreover, the uniformity improved dramatically, the PDI changed from 0.238 down to 0.044 , and the standard deviation in particle diameter was reduced from 154.8 to $38.13 \mathrm{~nm}$. Interestingly, when $2 \mathrm{wt} \%$ polyethylene glycol (PEG), a commonly used stabilizer, was added into the system, the standard deviation increased twofold, the PDI value increased to 0.3 from 0.044 , and the mean particle size was practically unchanged. This is clearly shown in DLS particle size distribution (Figure 3). As suggested in earlier work, applying ultrasound in multiphase systems can be used to stabilize and reduce the size of emulsions. ${ }^{46}$ In order to confirm the repeatability and uniformity of the results, the synthesis experiments were repeated 14 different times under identical conditions. For each experiment, mean diameters were calculated after 10 repeat measurements.

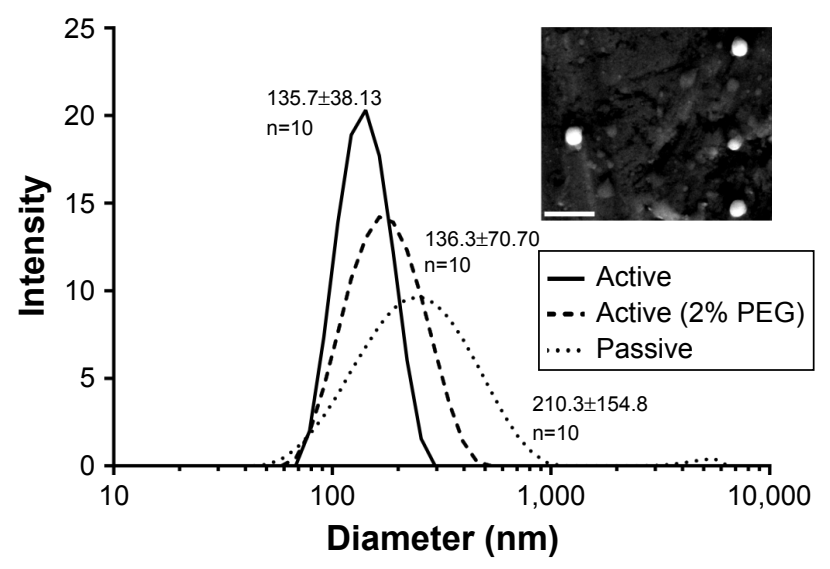

Figure 3 The effect of actuation on the particle diameter of budesonide - activation at $177.6 \mathrm{kHz}$ with the flow rate of $8 \mu \mathrm{L} /$ minute with a concentration of $0.2 \mathrm{mg} / \mathrm{mL}$ (results are based on 10 measurements).

Note: Inset: scanning electron micrograph of the particles (scale bar: $500 \mathrm{~nm}$ ).

Abbreviation: PEG, polyethylene glycol. 

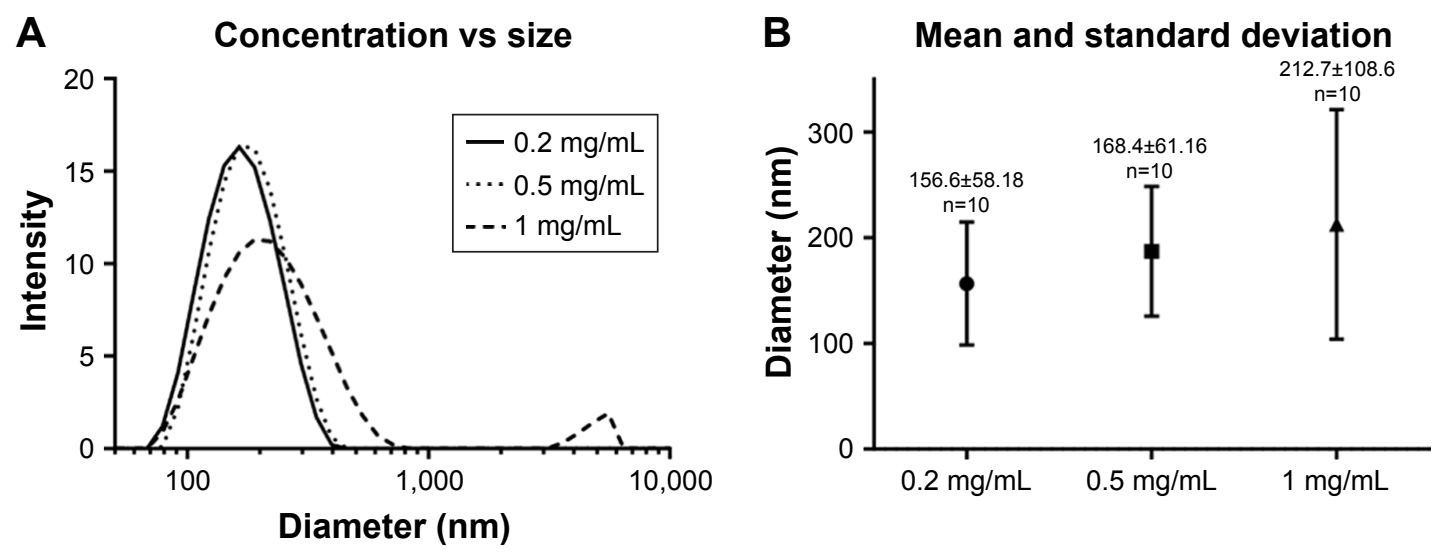

Figure 4 The effect of budesonide concentration on the mean size of nanoparticles.

Note: (A) DLS results and (B) mean diameter and standard deviations of the particle diameters as a function of the concentration (total flow rate: $20 \mu \mathrm{L} / \mathrm{minute}$ at I56.7 $\mathrm{kHz}$ actuation)

Abbreviation: DLS, dynamic light scattering.

The estimated mean diameter is $136.4 \mathrm{~nm}$ with a standard deviation of $1.06 \mathrm{~nm}$ for mean values, hence indicating that the results are highly repeatable.

Introduction of the active mixing during the LAS process enhanced the induction period, nucleation rate, and metastable zone width. As the antisolvent was rapidly mixed with the organic solvent for the solution to reach supercritical concentration, the insoluble drugs emerged from the solvent solution.

Many studies have shown the advantages of ultrasound in crystallization. For instance, ultrasound probe was used in a $\mathrm{T}$-junction channel to enhance the uniformity of particles. ${ }^{46-48}$ The effect of ultrasound in the crystallization process was investigated in a milli-fluidic device which employed a PZT plate vibrating beneath the mixing channel. ${ }^{49}$ Similarly, numerous studies of antisolvent crystallization were considered, ${ }^{50,51}$ where ultrasound was introduced from the bottom of the beakers while mixing was enhanced via diffusion or mechanical rotation of a stirrer. All of these have shown varying levels of improvement in the uniformity and size of particles across different studies.

A more recent study in $2016^{52}$ used ultrasound to reduce the diameter of budesonide-loaded solid lipid to 170-200 nm. However, it should be noted that this process requires an extremely long homogenization time and involves the use of complex solutions of Lipoid S100, S154, and budesonide. In contrast, our device homogenizes a continuous solution without emulsifiers at significantly shorter times. The mixing occurs almost instantaneously. The difference can be accounted for by the fact that ultrasound is integrated fully as a driving mechanism of mixing, rather than simply aiding the process; this enables a fast mixing time and a total elimination of surfactants from the process.
The device performance was investigated at concentrations ranging from 0.2 to $1 \mathrm{mg} / \mathrm{mL}$ under a fixed flow rate of $20 \mu \mathrm{L} /$ minute with a square-shaped membrane actuated at $156.7 \mathrm{kHz}$. As the concentration of the drugs increased by fivefold, the mean particle diameter was observed to change to $212.7 \mathrm{~nm}$, still offering a considerable improvement with respect to earlier studies. For 0.2 and $0.5 \mathrm{mg} / \mathrm{mL}$ concentrations, the nanoparticles continued to be synthesized at very high uniformity, which was compromised at higher concentrations, as shown in Figure 4. For this study, the drug was studied at low concentrations to eliminate the need for stabilizers and establish the smallest diameter particles that can be synthesized. It is worth noting that multiple mixers could be set in parallel to increase the yield of the nanodrugs.

Finally, the device performance was assessed at operational flow rates ranging from 10 to $50 \mu \mathrm{L} / \mathrm{min}$ (Figure 5). As the flow rates were increased, the average diameters varied

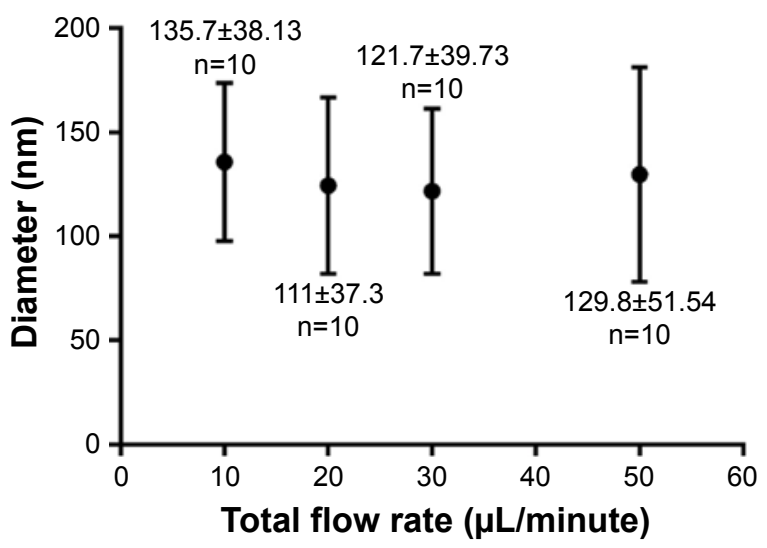

Figure 5 The effect of flow rate on the synthesized budesonide particles with the concentration of $0.2 \mathrm{mg} / \mathrm{mL}$ at a frequency of $177.6 \mathrm{kHz}$, for total flow rates 10,20 , 30 , and $50 \mu \mathrm{L} /$ minute $(\mathrm{n}=10)$. 


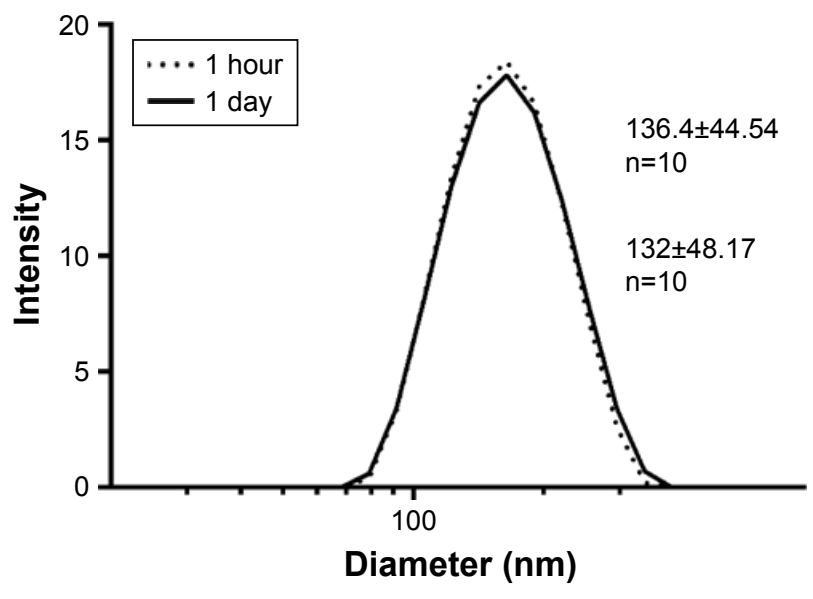

Figure 6 Stability of the budesonide particles. Note: Particle size distribution I hour and I day after synthesis.

between from $111 \mathrm{~nm}$ and $135.7 \mathrm{~nm}$, while the uniformity decreased slightly (standard deviation in the particle size distribution changed from 38.1 to $51.5 \mathrm{~nm}$ ). To demonstrate the stability of the particles produced with the method, DLS measurements were conducted 1 hour and 1 day after mixing. Figure 6 shows that despite lack of stabilizers, the particle size distribution remained constant and no coalescence was observed.

An acoustically enhanced microfluidic method was demonstrated to synthesize highly uniform budesonide nanoparticles with a mean diameter of $<150 \mathrm{~nm}$, reducing the size by 40 -fold in comparison to earlier works. This was achieved without adding any surfactants into the mixture, hence, significantly simplifying the process and eliminating undesirable complications, which are associated with additives.

\section{Disclosure}

The authors report no conflicts of interest in this work.

\section{References}

1. Liversidge EM, Liversidge GG. Drug nanoparticles: formulating poorly water-soluble compounds. Tox Path. 2008;36(1):43-48.

2. Junghanns JU, Muller RH. Nanocrystal technology, drug delivery and clinical applications. Int J Nanomedicine. 2008;3(3):295-309.

3. Rao S, Song Y, Peddie F, Evans AM. Particle size reduction to the nanometer range: a promising approach to improve buccal absorption of poorly water-soluble drugs. Int J Nanomedicine. 2011;6:1245-1251.

4. Sun J, Wang F, Sui Y, et al. Effect of particle size on solubility, dissolution rate, and oral bioavailability: evaluation using coenzyme $Q_{10}$ as naked nanocrystals. Int J Nanomedicine. 2012;7:5733-5744.

5. Hua M, Hua X. Polymer nanoparticles prepared by supercritical carbon dioxide for in vivo anti-cancer drug delivery. Nano Micro Lett. 2014;6: 20-23.

6. Chen X, Zhang JJ, Xuan J, Zhu JJ. Myoglobin/gold nanoparticles/ carbon spheres 3-D architecture for the fabrication of a novel biosensor. Nano Res. 2009;2(3):210-219.
7. Zhang L, Su T, He B, Gu Z. Self-assembly polyrotaxanes nanoparticles as carriers for anticancer drug methotrexate delivery. Nano Micro Lett. 2014;6(2):108-115.

8. Wu L, Cai X, Nelson K, et al. A green synthesis of carbon nanoparticle from honey for real-time photoacoustic imaging. Nano Res. 2013; 6(5):312-325.

9. Nan J, Chen Y, Li R, et al. Polymeric hydrogel nanocapsules: a thermo and $\mathrm{pH}$ dual-responsive carrier for sustained drug release. Nano Micro Let. 2014;6(3):200-208.

10. De Jong WH, Borm PJ. Drug delivery and nanoparticles: applications and hazards. Int J Nanomedicine. 2008;3(2):133-149.

11. Murthy SK. Nanoparticles in modern medicine: state of the art and future challenges. Int J Nanomedicine. 2007;2(2):129-141.

12. Onoue S, Yamada S, Chan HK. Nanodrugs: pharmacokinetics and safety. Int J Nanomedicine. 2014;9:1025-1037.

13. Pandey R, Sharma S, Khuller GK. Oral solid lipid nanoparticle-based antitubercular chemotherapy. Tuberculosis (Edinb). 2005;85(5-6): 415-420.

14. Heyder J, Gebhart J, Rudolf G, Schiller CF, Stahlhofen W. Deposition of particles in the human respiratory tract in the size range $0.005-15 \mu \mathrm{m}$. J Aerosol Sci. 1986;17(5):811-825.

15. Rauf A, Bhatnagar A, Sisodia SS, Khar RK, Ahmad FJ. Lungs deposition and pharmacokinetic study of submicron budesonide particles in Wistar rats intended for immediate effect in asthma. EXCLI J. 2017; 16:236-244.

16. Sung JC, Pulliam BL, Edwards DA. Nanoparticles or drug delivery to the lungs. Trends Biotechnol. 2007;25(12):563-570.

17. Duret C, Wauthoz N, Sebti T, Vanderbist F, Amighi K. New inhalationoptimized itraconazole nanoparticle-based dry powders for the treatment of invasive pulmonary aspergillosis. Int J Nanomedicine. 2012; 7: $5475-5489$.

18. Mansour HM, Rhee YS, Wu X. Nanomedicine in pulmonary delivery. Int J Nanomedicine. 2009;4:299-319.

19. Zarogoulidis P, Chatzaki E, Porpodis K, et al. Inhaled chemotherapy in lung cancer: future concept of nanomedicine. Int J Nanomedicine. 2012;7:1551-1572.

20. Shao Z, Shao J, Tan B, et al. Targeted lung cancer therapy: preparation and optimization of transferrin-decorated nanostructured lipid carriers as novel nanomedicine for co-delivery of anticancer drugs and DNA. Int J Nanomedicine. 2015;10:1223-1233.

21. Lee WH, Lee CY, Traini D, Young PM. Inhalation of nanoparticle-based drug for lung cancer treatment: advantages and challenges. Young Asian J Pharm Sci. 2015;10(6):481-489.

22. Manoj S, Manikanandan S, Kumaraguru AK. Nanoparticles: a new technology with wide applications. Res J Nanosci Nanotech. 2011; 1(1):1-11.

23. Thorat AA, Dalvi SV. Liquid antisolvent precipitation and stabilization of nanoparticles of poorly water soluble drugs in aqueous suspensions: Recent developments and future perspective. Chem Eng J. 2012;181-182:1-34.

24. Shah P. Use of nanotechnologies for drug delivery. MRS Bull. 2006; 31(11):894-899.

25. Sierra-Pallares J, Huddle T, García-Serna J, et al. Understanding bottom-up continuous hydrothermal synthesis of nanoparticles using empirical measurement and computational simulation. Nano Res. 2016; 9(11):3377-3387.

26. Nerowski A, Opitz J, Baraban L, Cuniberti G. Bottom-up synthesis of ultrathin straight platinum nanowires: electric field impact. Nano Res. 2013;6(5):303-311.

27. Al-Kassas R, Bansal M, Shaw J. Nanosizing techniques for improving bioavailability of drugs. J Control Release. 2017;260:202-212.

28. Kalani M, Yunus R. Application of supercritical antisolvent method in drug encapsulation: a review. Int J Nanomedicine. 2011;6:1429-1442.

29. Matteucci ME, Hotze MA, Johnston KP, Williams RO 3rd. Drug nanoparticles by antisolvent precipitation: mixing energy versus surfactant stabilization. Langmuir. 2006;22(21):8951-8959. 
30. Horn D, Rieger J. Organic nanoparticles in the aqueous phase-theory, experiment, and use. Angew Chem Int Ed Engl. 2001;40(23):4330-4361.

31. Gao L, Zhang D, Chen M. Drug nanocrystals for the formulation of poorly soluble drugs and its application as a potential drug delivery system. J Nanopart Res. 2008;10(5):845-862.

32. Phan HV, Coşkun MB, Şeşen M, Pandraud G, Neild A, Alan T. Vibrating membrane with discontinuities for rapid and efficient microfluidic mixing. Lab Chip. 2015;15(21):4206-4216.

33. Tattersfield AE, Harrison TW. Low-dose budesonide for asthma. Lancet. 2003;361(9363):1066-1067.

34. Godfrey S, Avital A, Rosler A, Mandelberg A, Uwyyed K. Nebulised budesonide in severe infantile asthma. Lancet. 1987;2(8563):851-852.

35. de Jongste JC, Duiverman EJ. Nebulised budesonide in severe childhood asthma. Lancet. 1989;1(8651):1388.

36. Turpeinen M, Pelkonen AS, Selroos O, Nikander K, Haahtela T. Continuous versus intermittent inhaled corticosteroid (budesonide) for mild persistent asthma in children - not too much, not too little. Thorax. 2012;67(2):100-102.

37. Jacobs C, Müller RH. Production and characterization of a budesonide nanosuspension for pulmonary administration. Pharm Res. 2002; 19(2):189-194.

38. Müller RH, Jacobs C, Kayser O. Nanosuspensions as particulate drug formulations in therapy. Rationale for development and what we can expect for the future. Adv Drug Deliv Rev. 2001;47(1):3-19.

39. Ali H, Weigmann B, Neurath MF, Collnot EM, Windbergs M, Lehr CM. Budesonide loaded nanoparticles with $\mathrm{pH}$-sensitive coating for improved mucosal targeting in mouse models of inflammatory bowel diseases. J Control Release. 2014;183:167-177.

40. Xu LM, Hu TT, Pu Y, et al. Preparation of high-performance ultrafine budesonide particles for pulmonary drug delivery. Chem Eng J. 2014;252:281-287.

41. Lindon JC, Trenter GE, Koppenaal D. Encyclopedia of Spectroscopy and Spectrometry. 2nd ed. Burlington: Elsevier; 2010

42. Hao JF, Fang XS, Zhou YF, et al. Development and optimization of solid lipid nanoparticle formulation for ophthalmic delivery of chloramphenicol using a Box-Behnken design. Int J Nanomedicine. 2011;6: 683-692.
43. Galton F, McAlister BA. The geometric mean, in vital and social statistics. Proc R Soc Lond. 1879;29:365-367.

44. Thøgersen A, Bonsak J, Fosli CH, Muntingh G. Size distributions of chemically synthesized Ag nanocrystals. J App Phys. 2011;110(4): 44306.

45. Saboti D, Maver U, Chan HK, Planinšek O. Novel budesonide particles for dry powder inhalation prepared using a microfluidic reactor coupled with ultrasonic spray freeze drying. J Pharm Sci. 2017;106(7): 1881-1888.

46. Kaiser A, Liu T, Richtering W, Schmidt AM. Magnetic capsules and pickering emulsions stabilized by core-shell particles. Langmuir. 2009;25(13):7335-7341.

47. Dalvi SV, Dave RN. Controlling particle size of a poorly water-soluble drug using ultrasound and stabilizers in antisolvent precipitation. Ind Eng Chem Res. 2009;48(16):7581-7593.

48. Pandey K, Chatte A, Dalvi S. Continuous production of aqueous suspensions of ultra-fine particles of curcumin using ultrasonically driven mixing device. Pharm Dev Technol. 2017;22:1-12.

49. Jamshidi R, Rossi D, Saffari N, et al. Investigation of the effect of ultrasound parameters on continuous sonocrystallization in a millifluidic device. Cryst Growth Des. 2016;16(8):4607-4619.

50. Lee J, Ashokkumar M, Kentish SE. Influence of mixing and ultrasound frequency on antisolvent crystallisation of sodium chloride. Ultrason Sonochem. 2014;21(1):60-68

51. Nii S, Takayanagi S. Growth and size control in anti-solvent crystallization of glycine with high frequency ultrasound. Ultrason Sonochem. 2014;21(3):1182-1186.

52. Esmaeili M, Aghajani M, Abbasalipourkabir R, Amani A. Budesonideloaded solid lipid nanoparticles for pulmonary delivery: preparation, optimization, and aerodynamic behaviour. Artif Cells Nanomed Biotechnol. 2016;44(8):1964-1971.
International Journal of Nanomedicine

\section{Publish your work in this journal}

The International Journal of Nanomedicine is an international, peerreviewed journal focusing on the application of nanotechnology in diagnostics, therapeutics, and drug delivery systems throughout the biomedical field. This journal is indexed on PubMed Central, MedLine, CAS, SciSearch $\AA$, Current Contents $₫ /$ Clinical Medicine,

\section{Dovepress}

Journal Citation Reports/Science Edition, EMBase, Scopus and the Elsevier Bibliographic databases. The manuscript management system is completely online and includes a very quick and fair peer-review system, which is all easy to use. Visit http://www.dovepress.com/ testimonials.php to read real quotes from published authors. 